\title{
Balloon aortic valvuloplasty of severe aortic stenosis in the era of TAVR
}

\author{
P. Kogoj ${ }^{1}$, T. Furlan' ${ }^{2}$, M. Bunc ${ }^{1}$ \\ ${ }^{1}$ Department of Cardiology, Division of Internal Medicine, University Medical Centre Ljubljana, Slovenia \\ ${ }^{2}$ Department of Internal Medicine, General Hospital Jesenice, Slovenia
}

Abstract

Introduction. Balloon aortic valvuloplasty (BAV) is a percutaneous treatment option for aortic stenosis (AS). Because of early restenosis and poor long-term survival, it is used as a bridge to surgery (SAVR) or transcatheter aortic valve replacement (TAVR), in hemodinamically unstable patients or patients that require urgent non-cardiac surgery.

Aim. The aim of this study was to evaluate all the BAV procedures performed in our centre in 2010 and to report our experience with BAV as a potential bridge to definitive therapy or as a palliative treatment.

Methods. We retrospectively analyzed all the patients who underwent percutaneous treatment of aortic stenosis in our institution between January and December 2010. We stratified our cohort into 3 groups: BAV as a bridge to TAVR or SAVR, BAV as a final therapy and TAVR without prior BAV. We evaluated patient characteristics, echocardiographic data and peri-procedural complications in each cohort. Survival was evaluated using Kaplan-Meier analysis.

Results. We included 86 high risk symptomatic patients with mean age of $82.2 \pm 5.0$ years and mean logistic EuroSCORE of $19.2 \pm 11.8 \%$. After BAV we observed a significant decrease in mean transvalvular gradient (from $43.8 \pm 14.4$ to $33.5 \pm 12.3 \mathrm{mmHg} ; \mathrm{p}<0.01$ ) and a significant increase in aortic valve area after BAV (from $0.6 \pm 0.2$ to $0.8 \pm 0.3 \mathrm{~cm}^{2} ; p<0.01$ ). Systolic pulmonary artery pressure, left ventricular ejection fraction and mitral regurgitation did not change significantly. Major intrahospital complications occurred in 5 patients $(6.5 \%)$, without any death related to the procedure. BAV as a bridge to TAVR had a better outcome compared with BAV alone.

Conclusion. BAV is a feasible and reasonably safe approach for temporary relief of symptoms, improvement of quality of life, decrease of surgical risk prior major non-cardiac surgery or as a bridge to surgical or transcatheter aortic valve implantation in severe aortic stenosis.

\section{Introduction}

alcific stenosis of the aortic valve (AS) is the most common acquired valve disorder in the Western world ${ }^{1}$. Surgical aortic valve replacement (SAVR) is considered the treatment of choice in patients with symptomatic AS regardless of $a^{2,3}$. The surgical risk in elderly patients with multiple comorbidities may be high and the presence of concomitant coronary artery disease (CAD) with the need for additional coronary artery bypass may duplicate the risk $^{4-6}$. Balloon aortic valvuloplasty (BAV) was introduced in 1986 as the first less invasive percutaneous treatment option of symptomatic $\mathrm{AS}^{7}$. Unfortunately, early restenosis of the dilated valve with symptoms recurrence and poor long-term survival limits the use of this procedure $^{8-12}$. Today BAV may be considered as a bridge to surgery or percutaneous aortic valve replacement (TAVR) in haemodynamic unstable patients or patients that require urgent major non-cardiac surgery. Balloon valvuloplasty may also be considered as a palliative measure in selected individual cases when surgery is contraindicated because of severe comorbidities and when TAVR is not an option ${ }^{13}$.

Percutaneous aortic valve treatment program started in our medical centre in 2008 as a part of preparation for TAVR program. BAV was not frequently used in our centre before. Since 2008 we performed over 330 BAV procedures and 110 TAVR implantations. The present study evaluated all the BAV procedures we performed in 2010 and reports our experience with BAV in symptomatic high risk patients with severe aortic stenosis, as a potential bridge to definitive therapy or as a palliative treatment.

\section{Methods}

\section{Patient population and selection}

We retrospectively analyzed all the patients who underwent percutaneous treatment of aortic stenosis in our institution between January and December 2010. We stratified our cohort into 3 groups according to the 
final treatment: BAV as a bridge to TAVR or SAVR, BAV as a final therapy (including repeated BAV) and TAVR without prior BAV.

Criteria for BAV and/or TAVR included:

- symptomatic aortic stenosis confirmed by transthoracic echocardiography with aortic valve area $<1 \mathrm{~cm}^{2}\left(<0.6 \mathrm{~cm}^{2} / \mathrm{m}^{2}\right)$,

- operative risk estimated by logistic EuroSCORE (European System of Cardiac Operative Risk Evaluation) > $15 \%$,

- contra-indication for surgery because of comorbid conditions; assessed and agreed to by both an independent cardiologist and a cardiovascular surgeon.

Criteria for BAV as a bridge to TAVR or SAVR included:

- hemodynamic unstable patient,

- prior urgent major non-cardiac surgery (for example patients with carcinoma),

- mitral regurgitation $(M R)>2+$,

- pulmonary hypertension due to combination of COPB and left ventricular failure.

\section{Echocardiographic data}

The screening before and after the percutaneous procedure included transthoracic echocardiography (TTE) recording of aortic stenosis severity, aortic valve regurgitation, left ventricular function, additional mitral regurgitation and pulmonary artery systolic pressure.

\section{BAV procedure}

The procedures were performed in our cardiac catheterisation laboratory in local anesthesia. Patients selected for BAV underwent diagnostic right and left cardiac catheterization. In case of coronary artery disease an angioplasty was performed before BAV in the same intervention. Heparin $(70 \mathrm{Ul} / \mathrm{kg}$ ) was administered after $10 \mathrm{~F}$ sheath insertion in the femoral artery and pig tail through $5 \mathrm{~F}$ sheet in radial artery. Aortic valve was crossed by LA1 catheter (Cordis) and J wire. J.wire was substituted for supportive Amplatz stiff wire (Abbott). Via percutaneous trans-femoral approach a balloon catheter (Zelos, OptiMed, $18-25 \mathrm{~mm} \times 4.0 \mathrm{~cm}$ ) was introduced and positioned across the stenotic aortic valve. We used undersized dilatation balloons according to TTE annulus diameter. Aortic valvuloplasty was performed with balloon inflation (25-30 ml, burst rate 4 ATM). Selection of the balloon inflation size was based on the combination of transthoracic echocardiography, baseline aortography, and alignment of the balloon to aortic annulus during valvuloplasty balloon expansion. To stabilize the balloon position across the valve, rapid ventricular pacing (180220 beats/min) was done during each inflation using temporary pacemaker electrode inserted in right ventricle through femoral vein. Before and after the valvuloplasty peak to peak pressure gradient was measure with pigtail catheters into aorta and left ventricle. The goal of the procedure was a reduction of the gradient by at least $50 \%$ and if necessary, the balloon inflation was repeated. In case of first BAV balloon under-sizing the second BAV was performed with the next, bigger sized balloon. Hemostasis was performed with vascular closure devices Pro- glide (Abbott vascular) or with direct manual compression of radial and femoral arterial puncture site ad once and after 3-4 hours, respectively.

\section{TAVR procedure}

The selection of TAVR candidates was done according to Edwards recommendations. All the patients were refused for classic AVR. Indication for TAVR was also conditioned by TAV availability. We used Edwards-Sapien devices. Sizing was done by combination of CTA and TEE. Implantations were performed in coronary cathlab in sterile regimen. TAVI team members (anaesthetist, cardiovascular surgeon, interventional cardiologists, ECHO specialists) participated in the patients selection. Patients were operated in general anesthesia. Both trans-apical and trans-femoral TAVR approaches were utilized.

\section{Data collection and follow up}

Baseline clinical data were retrospectively collected from the medical records. Logistic EuroSCORE was calculated for all patients based on baseline variables before the procedure. All clinically relevant baseline and follow-up variables as well as peri-procedural complication were prospectively entered into a dedicated database. In-hospital follow-up consisted of vital parameters, complete blood count and assessment of renal function, review of arterial puncture site and TTE within few days after BAV. Clinical and echocardiographic follow up was planned at 3 to 6 months after BAV and obtained from chart review.

Major peri-procedural adverse events were defined as peri-procedural death from any cause, myocardial infarction, severe acute aortic regurgitation, stroke, cardiac tamponade, cardiogenic shock, aortic dissection, major vascular complications, urgent or emergency conversion to surgery or permanent pacemaker requirement. Acute renal impairment, myocardial infarction, stroke, vascular complications and major bleeding were defined according to the Valve Academic Research Consortium proposed criteria (VARC) ${ }^{14}$.

\section{Statistical analysis}

Qualitative variables were expressed as percentages and quantitative variables were expressed as mean \pm standard deviation. Comparison of continuous variables was performed with the analysis of variance and Student's paired t-test, as appropriate. The $\chi 2$ test was used to compare qualitative variables. Survival rates were presented as Kaplan-Meier curves, and the log-rank test was used for comparison. Differences were considered statistically significant at $\mathrm{P}<0.05$. All data were processed using the Statistical Package for Social Sciences, version 17.0 (SPSS, Inc., Chicago, Illinois).

\section{Results}

The cohort included 86 consecutive patients who underwent percutaneous treatment of severe aortic stenosis in 2010 in our institution. BAV was performed in 77 patients $(89.5 \%)$, followed by TAVR in 19 patients (22.1 
$\%)$ and SAVR in one patient (1.2 \%). Nine patients (10.5 $\%)$ underwent TAVR without previous BAV procedure.

\section{Baseline characteristics}

The baseline characteristics of all patients are displayed in the Table 1 . They all had symptomatic severe aortic stenosis with mean AVA of $0.6 \pm 0.2 \mathrm{~cm}^{2}$ and were at high risk for surgical valve replacement. All the patients were older than 80 years with the mean age of $82.2 \pm 5.0$ years, mean logistic EuroSCORE of $19.2 \pm 11.8$ and 54 $(62.8 \%)$ of them were presenting in New Your Heart Association (NYHA) class III/IV. Common comorbidities included hypertension (72.1\%), coronary artery disease (41.9\%) with previous coronary artery bypass grafting (14 $\%)$, chronic kidney disease (34.9\%), chronic obstructive pulmonary disease (26.7\%) and diabetes (25.6\%).

\section{Indications for BAV}

Table 1. Baseline characteristics of the overall population.

\begin{tabular}{|c|c|}
\hline Variables & $\begin{array}{l}\text { Overall } \\
\text { population } \\
(n=86)\end{array}$ \\
\hline Age, y & $82.2 \pm 5.0$ \\
\hline Men, $\mathrm{n}(\%)$ & $28(32.6)$ \\
\hline Logisitic EuroSCORE & $19.2 \pm 11.8$ \\
\hline Cardiogenic shock, n (\%) & $1(1.2)$ \\
\hline NYHA III and IV, n (\%) & $54(62.8)$ \\
\hline Diabetes, n (\%) & $22(25.6)$ \\
\hline Hypertension, n (\%) & $62(72.1)$ \\
\hline Hyperlipidemia, n (\%) & $12(14.0)$ \\
\hline $\begin{array}{l}\text { Coronary artery disease, } \mathrm{n} \mathrm{( \% )} \\
\text { (stenosis more than 70\%) }\end{array}$ & 36 (41.9) \\
\hline Chronic obstructive pulmonary disease, $\mathrm{n}(\%)$ & $23(26.7)$ \\
\hline Chronic renal failure, $\mathrm{n}(\%)$ & 30 (34.9) \\
\hline Prior CVI, n (\%) & $14(16.3)$ \\
\hline Atrial fibrillation, $\mathrm{n}(\%)$ & $43(50)$ \\
\hline Peripheral artery disease, $\mathrm{n}(\%)$ & $9(10.5)$ \\
\hline Prior coronary artery bypass grafting, $\mathrm{n}(\%)$ & $12(14.0)$ \\
\hline Prior percutaneous coronary intervention, $\mathrm{n}(\%)$ & $17(19.8)$ \\
\hline Prior myocardial infarction, $\mathrm{n}(\%)$ & $6(7.0)$ \\
\hline Neoplasma, $\mathrm{n}(\%)$ & $16(18.6)$ \\
\hline Porcelain aorta, $\mathrm{n}(\%)$ & $1(1.2)$ \\
\hline Mean aortic gradient, $\mathrm{mm} \mathrm{Hg}$ & $44.2 \pm 13.7$ \\
\hline Baseline AVA, $\mathrm{cm}^{2}$ & $0.6 \pm 0.2$ \\
\hline AR grade & $1-2 / 4$ \\
\hline LVEF, \% & $52.0 \pm 14.4$ \\
\hline $\mathrm{BAV}$ and $\mathrm{PCl}$ as a single procedure & $14(16.3)$ \\
\hline BAV prior non-cardiac surgery & $3(3.5)$ \\
\hline
\end{tabular}

Results presented as mean \pm standard deviation/ number (\%) Abbreviations: NYHA: New York Heart Association; CVI: cerebrovascular insult; AVA: aortic valve area; AR: aortic regurgitation; LVEF: left ventricular ejection fraction; $\mathrm{BAV}$ : balloon aortic valvuloplasty; $\mathrm{PCl}$ : percutaneous coronary intervention

Indications for BAV included symptoms relief (47; $61.0 \%)$, bridge to SAVR $(1 ; 1.3 \%)$ or TAVR $(25 ; 32.5 \%)$,

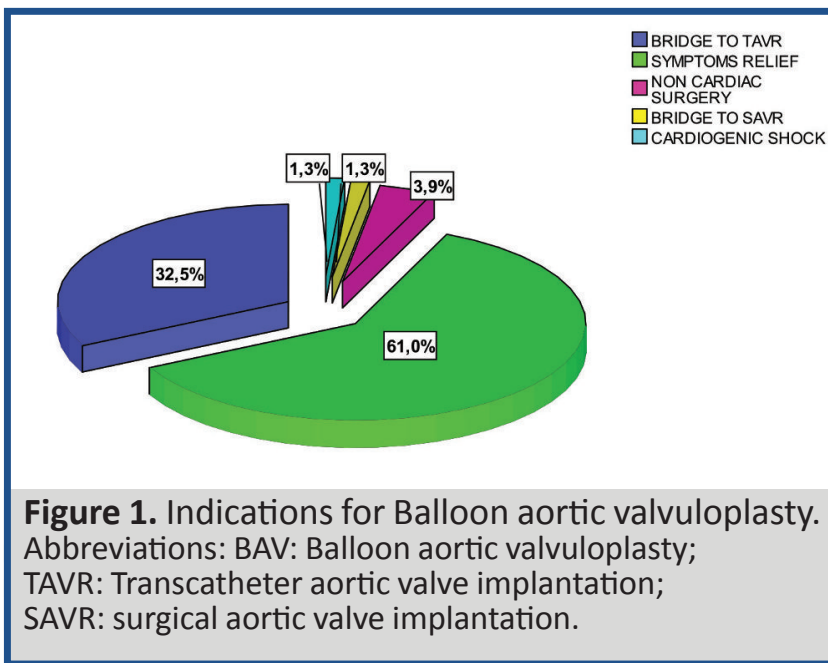

hemodynamic unstable patients in cardiogenic shock or sepsis $(1 ; 1.3 \%)$, oncologic patients prior urgent noncardiac surgery $(3 ; 3.9 \%)$ (Figure 1$)$.

\section{Hemodynamic results}

After BAV we observed a significant decrease in mean trans-valvular gradient (from $43.8 \pm 14.4$ to 33.5 $\pm 12.3 \mathrm{~mm} \mathrm{Hg} ; \mathrm{p}<0.01$ ) and an significant increase in aortic valve area (from $0.6 \pm 0.2$ to $0.8 \pm 0.3 \mathrm{~cm}^{2} ; \mathrm{p}<$ 0.01 ). Procedural success was achieved in $73 \%$ of patients (reduction of peak to peak gradient on half), however $84 \%$ of patients improved clinically with reduction of pulmonary and right side congestion. Systolic pulmonary artery pressure, left ventricular ejection fraction and mitral regurgitation did not significantly change after BAV.

Table 2. Intrahospital complications after BAV.

\begin{tabular}{|l|l|}
\hline Variables & $\begin{array}{l}\text { Overall population BAV } \\
(\mathrm{n}=77)\end{array}$ \\
\hline Overall major complication, $\mathrm{n}(\%)$ & $5(6.5 \%)$ \\
\hline Death for any causes, $\mathrm{n}(\%)$ & $1(1.3 \%)$ \\
\hline $\begin{array}{l}\text { Vascular complications, } \mathrm{n}(\%) \\
\text { Major } \\
\text { Minor }\end{array}$ & $\begin{array}{l}4(5.3 \%) \\
11(14.3 \%)\end{array}$ \\
\hline Myocardial infarction, $\mathrm{n}(\%)$ & $1(1.3 \%)$ \\
\hline Acute renal injury, $\mathrm{n}(\%)$ & $3(4.0 \%)$ \\
\hline $\begin{array}{l}\text { lodinated contrast induced } \\
\text { thyrotoxicosis, } \mathrm{n}(\%)\end{array}$ & $1(1.3 \%)$ \\
\hline
\end{tabular}

Abbreviations: BAV: balloon aortic valvuloplasty

\section{Procedural complications}

Complications related to BAV are summarized in Table 2. Major intra-hospital complications occurred in 5 patients (6.5\%). We did not observe any death related to the BAV procedure. One patient died few days after BAV because of cardiogenic shock developed before the procedure. Major vascular complication occurred in 4 patients (5.2\%) where we observed pseudo-aneurysm leading to unplanned surgical intervention. Other vascular complications were classified as minor in 11 pa- 
Table 3. Baseline characteristics according to the treatment after BAV in comparison to patients who underwent TAVR without previous BAV.

\begin{tabular}{|c|c|c|c|c|}
\hline Variables & BAV & TAVR & TAVR only & $p$ \\
\hline $\mathrm{N}$ & $57(66.3)$ & $19(22.0)$ & $9(10.5)$ & \\
\hline Age, y & $81.9 \pm 5.4$ & $83.1 \pm 4.0$ & $82.2 \pm 4.9$ & 0.65 \\
\hline Men, $\mathrm{n}(\%)$ & $21(36.8)$ & $3(15.8)$ & $4(44.4)$ & 0.18 \\
\hline Logisitic EuroSCORE, \% & $20.9+13.5$ & $17.2+7.4$ & $13.8+6.8$ & 0.18 \\
\hline $\mathrm{BAV}$ and $\mathrm{PCl}, \mathrm{n}(\%)$ (single procedure) & $9(15.8)$ & $5(26.3)$ & 0 & 0.21 \\
\hline NYHA, n & 3.1 & 2.9 & 2.2 & $<0.01$ \\
\hline NYHA III and IV, n (\%) & $39(68.4)$ & $12(63.2)$ & $2(22.2)$ & 0.03 \\
\hline Diabetes, $\mathrm{n}(\%)$ & $11(19.3)$ & $7(36.8)$ & $3(33.3)$ & 0.25 \\
\hline Hypertension, n (\%) & $36(63.2)$ & $18(94.7)$ & $7(77.8)$ & 0.03 \\
\hline Hyperlipidemia, n (\%) & $6(10.5)$ & $6(31.6)$ & 0 & 0.03 \\
\hline Coronary artery disease, $\mathrm{n}(\%)$ & $22(38.6)$ & $10(52.6)$ & $4(44.4)$ & 0.56 \\
\hline COPD, n (\%) & $16(28.1)$ & $5(26.3)$ & $2(22.2)$ & 0.93 \\
\hline Chronic renal failure, $\mathrm{n}(\%)$ & $22(38.6)$ & $7(36.8)$ & $1(11.1)$ & 0.27 \\
\hline Stroke, $\mathrm{n}(\%)$ & $10(17.5)$ & $2(10.5)$ & $1(11.1)$ & 0.71 \\
\hline Atrial fibrillation, $\mathrm{n}(\%)$ & $28(49.1)$ & $10(52.6)$ & $4(44.4)$ & 0.91 \\
\hline PAOD, n (\%) & $9(15.8)$ & 0 & 0 & 0.08 \\
\hline CABG, $\mathrm{n}(\%)$ & $8(14.0)$ & $3(15.8)$ & $1(11.1)$ & 0.95 \\
\hline Previous $\mathrm{PCl}, \mathrm{n}(\%)$ & $12(21.1)$ & $3(15.8)$ & $2(22.2)$ & 0.87 \\
\hline Previous myocardial infarction, $\mathrm{n}(\%)$ & $6(10.5)$ & 0 & 0 & 0.21 \\
\hline Cancer, n (\%) & $11(19.3)$ & $3(15.8)$ & $1(11.1)$ & 0.81 \\
\hline Porcelain aorta, n (\%) & 0 & $1(5.3)$ & 0 & 0.17 \\
\hline $\begin{array}{l}\text { Mean gradient, } \mathrm{mm} \mathrm{Hg} \\
\text { before procedure }\end{array}$ & $46.5 \pm 14.3$ & $35.7 \pm 10.5$ & $42.6 \pm 8.8$ & 0.11 \\
\hline $\begin{array}{l}\text { Mean gradient , } \mathrm{mmHg} \\
\text { after procedure }\end{array}$ & $35.5 \pm 13.8$ & $40.0 \pm 14.2$ & $11.5 \pm 2.1$ & $\begin{array}{l}0.03 \\
* 0.51 \\
\end{array}$ \\
\hline AVA before procedure, $\mathrm{cm}^{2}$ & $0.6 \pm 0.2$ & $0.6 \pm 0.2$ & $0.7 \pm 0.2$ & 0.11 \\
\hline AVA after procedure, $\mathrm{cm}^{2}$ & $0.7 \pm 0.2$ & $0.8 \pm 0.1$ & $1.6 \pm 0.2$ & $\begin{array}{l}<0.01 \\
* 0.70\end{array}$ \\
\hline LVEF, \% & $52.9 \pm 14.9$ & $48.0 \pm 15.1$ & $51.7 \pm 2.9$ & 0.63 \\
\hline Pulmonary hypertension, $\mathrm{mmHg}$ & $50.5 \pm 16.5$ & $43.5 \pm 7.5$ & $44.3 \pm 7.5$ & 0.36 \\
\hline Creatinin baseline, $\mu \mathrm{mol} / \mathrm{I}$ & $118.8 \pm 76.1$ & $103.9 \pm 33.5$ & $63.0 \pm 25.7$ & 0.06 \\
\hline NTproBNP baseline, ng/l & $9784.1 \pm 10271.4$ & $7255.6 \pm 11347.9$ & $2477,6 \pm 2554.5$ & 0.33 \\
\hline
\end{tabular}

Results presented as mean \pm standard deviation/ number (\%).

* $p$ value related to the comparison of the group that underwent BAV versus the group that underwent TAVR after BAV.

Abbreviations: TAVR: transcatheter aortic valve replacement; TAVR only: TAVR patients without prior BAV; PCI: percutaneous coronary intervention; NYHA: New York Heart Association; COPD: chronic obstructive pulmonary disease; PAOD: peripheral arterial occlusive disease; AVA: aortic valve area; LVEF: left ventricular ejection fraction.

tients $(14.3 \%)$ - need for blood transfusion and pseudoaneurysm ( 2 cases) with no need for vascular surgery. In one patient we observed iodinated contrast induced thyrotoxicosis which was successfully treated with thyreo-static drugs. The most common complication was related to vascular access site. Acute myocardial infarction occurred in one case (1.3\%) and acute renal injury in 3 cases ( $4 \%$ ). We did not observe severe aortic regurgitation, stroke, permanent pacemaker requirement, tamponade or anular rupture in our patients.

\section{Follow up and mortality}

Patients left hospital $13.4 \pm 10.8$ days after BAV. $92 \%$ of the patients were in follow up of $16.1 \pm 15.0$ months. Within this period 20 patients were finally bridged to definitive therapy [TAVR $n=19(22.1 \%)$ and SAVR $n=1$
(1.2\%)] and 66 (76.7\%) unsuitable for definitive therapy remained on medical therapy alone. In four patients $B A V$ was repeated once and in two patients BAV twice during the follow up period. In 14 cases with concomitant coronary artery disease we performed BAV and $\mathrm{PCI}$ in a single procedure with no increase in peri-procedural complication rate. In 3 oncologic patients with severe aortic stenosis that required a major abdominal and gynecology surgery BAV and in one case BAV and $\mathrm{PCI}$ was done prior surgery. With angioplasty and BAV we achieved a good coronary artery flow and an increase in aortic valve area without any peri-procedural complications. Successful non-cardiac surgery was consecutively done in all the 3 cases $^{15}$.

Baseline characteristics of patients according to final treatment after BAV are presented in Table 3 and compared with the characteristics of patients who underwent 
TAVR without previous BAV procedure. Overall, patients who underwent TAVR without previous BAV were less symptomatic and presented in lower NYHA functional class. There were no differences in AVA, mean trans-aortic gradient, pulmonary artery arterial pressure and mitral regurgitation severity between groups (Table 3).

Long-term follow-up was evaluated using KaplanMeier analysis, and survival curves according to treatment after BAV are shown in Figure 2. Patient bridged to TAVR had better outcomes compared with those treated by single BAV ( $p<0.01)$. There was no significant impact on survival in patients where BAV was performed more than once and in patients where TAVR was performed without previous BAV (Figure 2-4).

\section{Discussion}

BAV was originally proposed as an alternative to SAVR for severe symptomatic AS, but it was rapidly neglected secondary to high restenosis rates and no impact on survival. In the TAVR era BAV was reported to be inferior to TAVR and comparable to medical therapy in terms of survival (16). We can confirm that single BAV, without bridge to TAVR/SAVR is associated to bad outcome. However, BAV might be a reasonable approach to offer symptomatic relief and improvement of the quality of life in selected high risk, comorbid patients and patients with short life expectancy. Current guidelines support this statement (recommendation Class IIb) and suggest this per-cutaneous treatment also as a bridge to TAVR/SAVR in hemodynamic unstable patients or in patients who require urgent major non-cardiac surgery $(2,17)$.

\section{$\mathrm{BAV}$ as a definition therapy}

In our cohort of very high risk patients with aortic stenosis, BAV was associated with statistically significant hemodynamic results with significant decrease of mean aortic gradient in BAV, BAV-TAVR and TAVR group from: $46.5 \pm 14.3 \mathrm{mmHg}, 35.7 \pm 10.5 \mathrm{mmHg}, 42.6 \pm 8.8$ $\mathrm{mmHg}$ to $35.5 \pm 13.8 \mathrm{mmHg}, 40.0 \pm 14.2 \mathrm{mmHg}$ and $11.5 \pm 2.1$, respectively. The major indication for BAV remains symptomatic relief (61.0\%) and due to low major complication rate $(6.5 \%)$ it is a reasonable approach. Vascular access site complications remain the principal procedural limitation but with the development of the technique over the years and with the use of vascular closure devices we may reduce the complication rate. In contrast to our experience, Eltchaninoff et al., reported an increase of aortic valve area $>1 \mathrm{~cm}^{2}$ after BAV in most of the patients, recently (18). From this perspective, BAV may become a valid therapeutic option for high risk patients refused for surgery and unsuitable for TAVR. In our case AVA improved from $0.6 \pm 0.2$ to $0.8 \pm 0.3 \mathrm{~cm}^{2} ; \mathrm{p}<$ 0.01 . One of the reasons for less effective BAV comparing to the results published by Eltchaninoff et al. might be our conservative strategy. Namely, BAV is rather palliative procedure, therefore we were using undersized balloons for non-aggressive BAV. The rate of important AR and annulus damage is therefore zero. Most frequently we used BAV balloon of dimensions $20 \mathrm{~mm}, 23 \mathrm{~mm}$ and 25 $\mathrm{mm}$ in $78 \%, 18 \%$ and $4 \%$, respectively.

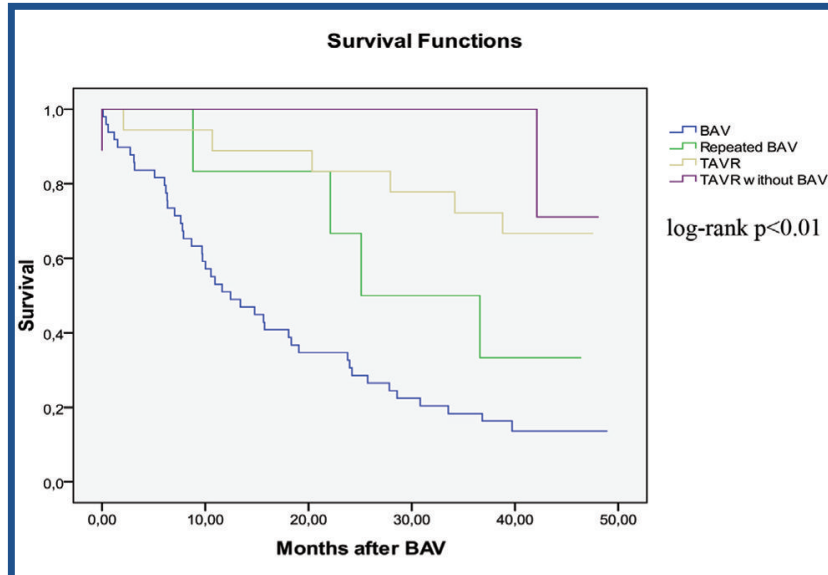

Figure 2. Kaplan-Meier estimated survival according to the percutaneous treatment of aortic stenosis. Abbreviations: BAV: balloon aortic valvuloplasty; TAVR: transcatheter aortic valve replacement; TAVR only: TAVR patients without prior BAV

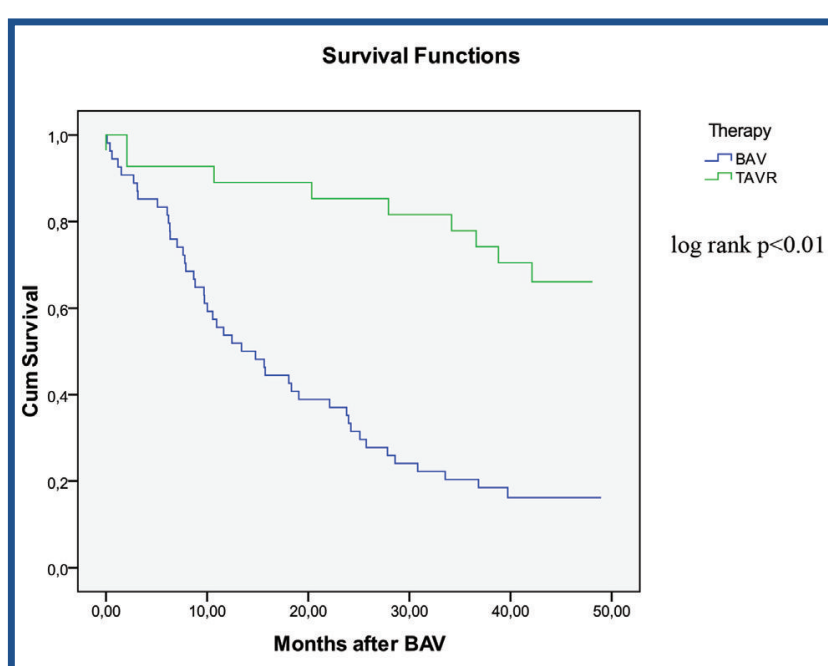

Figure 3. Kaplan-Meier estimated survival in BAV patients versus TAVR patients. Abbreviations: BAV: balloon aortic valvuloplasty; TAVR: transcatheter aortic valve replacement.

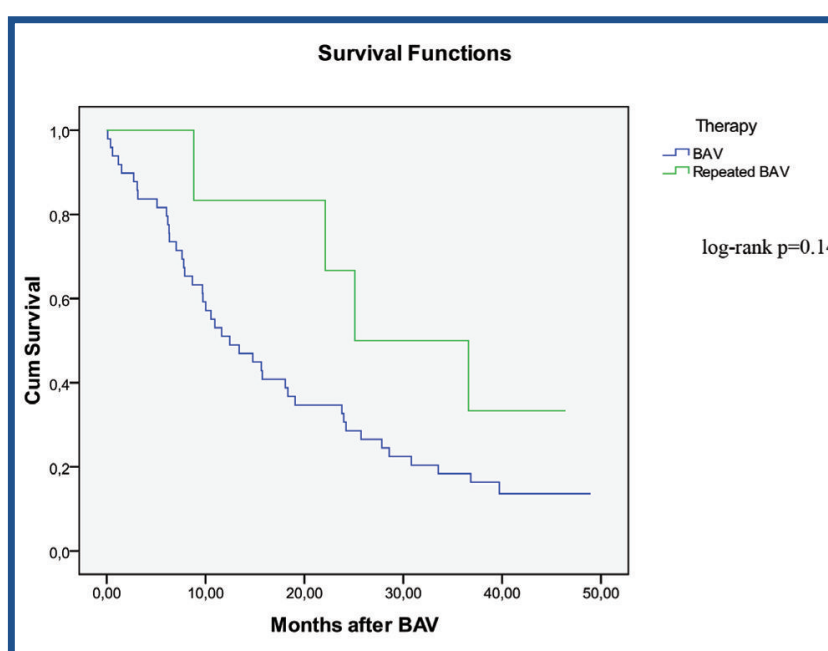

Figure 4. Kaplan-Meier estimated survival in patients who underwent a single BAV comparing to patients who underwent more than one BAV.

Abbreviations: BAV: balloon aortic valvuloplasty. 


\section{BAV as a bridge to TAVR/SARV}

Soon after BAV we often observe an improvement of hemodynamic conditions; an increase of cardiac output, a reduction of pulmonary pressure and improvement of other heart failure clinical presentations (16). As a consequence BAV is recommended as a bridge to TAVR/SAVR in hemodynamic instable patients suffering from a potentially reversible acute state (for example sepsis or cardiogenic shock) (13). In our cohort we performed BAV in one patient $(1.3 \%)$ who presented in cardiogenic shock but despite the successful procedure he died because of the underlying disease. BAV may be also performed in patients who are good candidates for TAVR but per-cutaneous valve implantation is not available because of the economical and organisational issues. In potential TAVR candidates BAV can also serve as a diagnostic measure, which can help define the final therapeutic option: BAV or TAVR. In particular it can be used to asses the potential impact of left ventricular afterload reduction on improvement of low left ventricular ejection fraction, severe pulmonary hypertension and mitral regurgitation improvement.

\section{BAV in cancer patients}

We performed BAV in three oncologic patients that underwent major abdominal surgery soon after the percutaneous aortic procedure. Successful BAV and hemodynamic improvement should decrease the risk for abdominal surgery. In our experience the surgery was performed without cardiovascular complications. In selected high risk carcinoma patients BAV may be used as bridge to TAVI which can be done if there is no carcinoma relapse one year after non-cardiac surgery.

\section{Long-term outcome after BAV}

According to the results of PARTNER trial (16) we demonstrated that $\mathrm{BAV}$ remain a palliative measure, with no impact on long-term survival. The survival in patients bridged to TAVR after BAV was significantly higher than in those treated with BAV alone. In patients where BAV was performed more than once in comparison to single BAV we observe a trend of better outcome that is not statistically significant. We can speculate that early restenosis of the dilated aortic valve and consecutive relatively rapid re-increase in left ventricular afterload prevent ventricular reverse remodelling and exhibit the positive impact on the left ventricle we achieved early after BAV. With prevention of restenosis we may find a way to improve the outcome after BAV but until now no treatment strategy succeed in this attempt (20).

\section{Conclusion}

In conclusion, BAV is a feasible and reasonably safe approach to offer temporary relief in selected high risk patients with symptomatic severe aortic. BAV can be utilized as a part of a complex to improve the quality of life, decrease the surgical risk for major non-cardiac surgery or as a bridge to surgical or trans-catheter aortic valve implantation. BAV can also be used to select the best further treatment strategy (TAVR/SAVR or medical therapy) in patients with impaired LV function, pulmonary hypertension and important mitral regurgitation.

\section{References}

1. Rajamannan NM, Bonow RO, Rahimtoola SH. Calcific aortic stenosis: An update. Nat Clin Pract Cardiovasc Med 2007;4:254-262.

2. Vahanian A, Baumgartner H, Bax J, et al; Task Force on the Management of Valvular Hearth Disease of the European Society of Cardiology; ESC Committee for Practice Guidelines. Guidelines on the management of valvular heart disease: The Task Force on the Management of Valvular Heart Disease of the European Society of Cardiology. Eur Heart J 2007;28:230-268.

3. Bonow RO, Carabello BA, Kanu C, et al. ACC/AHA 2006 guidelines for the management of patients with valvular heart disease: $A$ report of the American College of Cardiology/American Heart Association Task Force on Practice Guidelines: Developed in collaboration with the Society of Cardiovascular Anesthesiologists: Endorsed by the Society for Cardiovascular Angiography and Interventions and the Society of Thoracic Surgeons. Circulation 2006;114:e84-e231.

4. lung B, Cachier A, Baron $G$, et al. Decision-making in elderly patients with severe aortic stenosis: Why are so many denied surgery? Eur Heart J 2005;26:2714-2720.

5. Cribier A, Sabin T, Saoudi N, et al. Percutaneous transluminal valvuloplasty of acquired aortic stenosis in elderly patients: An alternative to valve replacement? Lancet 1986;1:63-67.

6. McKay RG, Safian RD, Lock JE, et al. Balloon dilatation of calcific aortic stenosis in elderly patients: Post mortem, intraoperative, and percutaneous valvuloplasty studies. Circulation 1986;74:119-125.

7. Zimrin DA, Reyes PA, Miller KL, et al. Heart failure and "inoperable" aortic stenosis: Staged balloon aortic valvuloplasty and aortic valve bypass. Congest Heart Fail 2008;14:211-213.

8. Kitamura H, Doi T, Okabayashi H, et al. Percutaneous transluminal balloon aortic valvuloplasty with a small balloon as a bridge to surgery for severe aortic stenosis in an 83-year-old patient. Jpn J Thorac Cardiovasc Surg 2003;51:562-564.

9. Dauterman KW, Michaels AD, Ports TA. Is there any indication for aortic valvuloplasty in the elderly? Am J Geriatr Cardiol 2003;12:190-196.

10. Smedira NG, Ports TA, Merrick SH, Rankin JS. Balloon aortic valvuloplasty as a bridge to aortic valve replacement in critically ill patients. Ann Thorac Surg 1993;55:914-916.

11. Pena HP, Teixeira SL, Mandil A, et al. Aortic balloon valvuloplasty as a bridge to definitive therapy of severe aortic stenosis. Arq Bras Cardiol 1997;69:55-57.

12. Vahanian A, Alfieri O, Al-Atta N, et al. Transchateter valve implantation for patients with aortic stenosis: A position statement from the European Association of Cardio-Thoracic Surgery (EACTS) and the European Society of Cardiology (ESC), in collaboration with the European Association of Percutaneous Cardiovascular Interventions (EAPCI). Eurolnterv 2008;4:193-199.

13. Vahanian A, Alfieri $O$, Andreotti F, et al. Guidelines on the management of valvular heart disease (version 2012): the Joint Task Force on the Management of Valvular Heart Disease of the European Society of Cardiology (ESC) and the European Association for Cardio-Thoracic Surgery (EACTS). Eur J Cardiothorac Surg 2012;42(4):1-44.

14. Leon MB, Piazza N, Nikolsky E, et al. Standardized endpoint definitions for transcatheter aortic valve implantation clinical trials: a consensus report from the Valve Academic Research Consortium. Eur Heart J 2011;32(2):205-17.

15. Kogoj P, Devjak R, Bunc M. Balloon aortic valvuloplasty (BAV) as a bridge to aortic valve replacement in cancer patients who require urgent non-cardiac surgery. Radiol Oncol 2014;48(1):62-6.

16. Leon MB, Smith CR, Mack M, et al. Transcatheter aortic-valve implantation for aortic stenosis in patients who cannot undergo surgery. N Engl J Med 2010;363(17):1597-607.

17. Nkomo VT, Gardin JM, Skelton TN, et al. Burden of valvular heart diseases: A population-based study. Lancet 2006;368:1005-1011.

18. Eltchaninoff H, Durand E, Borz B, et al. Balloon aortic valvuloplasty in the era of transcatheter aortic valve replacement: acute and long-term outcomes. Am Heart J 2014;167(2):235-40.

19. Kogoj P, Music S, Lakic N, et al. Balloon Aortic Valvuloplasty In Severe Aortic Stenosis And Prevention Of Restenosis In Elderly. J Am Coll Cardiol 2012;60:17. 